\title{
Alpha decay of Europium isotopes
}

\author{
O A P Tavares and E L Medeiros ${ }^{1}$ \\ Centro Brasileiro de Pesquisas Físicas - CBPF/MCT \\ Rua Dr. Xavier Sigaud 150, 22290-180 Rio de Janeiro-RJ, Brazil
}

\begin{abstract}
Pioneer experimental indication of an extremely low alpha activity due to ${ }^{151} \mathrm{Eu}$ isotope (equivalent to $\sim 10-70$ disintegrations/h-kg of ${ }^{\text {nat }} \mathrm{Eu}$ ) is discussed in the framework of a simple, one-parameter model to alpha transitions developed by us recently. The present evaluated alpha activity is found equivalent to $\sim 18$ disintegrations/h-kg, in excellent agreement with the observed decay rate. Partial alpha decay half-life evaluations for ${ }^{147-153} \mathrm{Eu}$ isotopes are also reported.
\end{abstract}

PACS: 23.60.+e

Keywords: alpha decay, Europium isotopes, half-life evaluations

\footnotetext{
${ }^{1}$ Author to whom correspondence should be addressed, emil@cbpf.br
} 
The Europium isotopes $(Z=63)$ of mass number from 130 up to 153 do exhibit the necessary requirement to disintegrate by alpha particle emission, i.e. a positive $Q_{\alpha^{-}}$ value [1]. However, for most of them the extremely high decay rates by the radioactive process of electron capture and, to a lesser extent, the $\beta^{-}$-decay process, make alpha decay accessible to only a few isotopes, namely, those of mass number 147, 148, 150, and the naturally occurring ${ }^{151} \mathrm{Eu}$ isotope (47.81\% of isotopic abundance). In addition, the very low $Q_{\alpha}$-value $(295 \mathrm{keV})$ for the other naturally occurring europium isotope ( ${ }^{153} \mathrm{Eu}$, $52.19 \%$ ) hinders any attempt to observe alpha decay from this isotope for its half-life is estimated to be tremendously greater than $\sim 10^{20} \mathrm{yr}$ (see below). Although alpha decay of the artificially produced ${ }^{147,148} \mathrm{Eu}$ isotopes has been established much before [2], it was just very recently that a collaboration research group from Kiev-Kharkov-BeijingRoma-Assergi observed for the first time an alpha activity assigned to ${ }^{151} \mathrm{Eu}$ isotope with an estimated half-life of $\left(5_{-3}^{+11}\right) \times 10^{18} \mathrm{yr}[3,4]$. The experiment was conducted deep underground at the Gran Sasso National Laboratories (INFN, Assergi, Italy), and all its technical details and data analyses can be appreciated in $[4,5]$. Here we report on partial alpha decay half-life evaluations for the ${ }^{147-153} \mathrm{Eu}$ isotopes and compare these results with available measured data. To this aim, use is made of a recent semiempirical approach to alpha decay we developed [6-9] motivated by the discovery of a rare alpha activity in natural bismuth [10].

The alpha transition from the ground state of ${ }^{151} \mathrm{Eu}$ isotope to the daughter ${ }^{147} \mathrm{Pm}$ can proceed mainly in two ways, namely, $i$ ) to the first excited state of the daughter at $91.1 \mathrm{keV}$ excitation with a $Q_{\alpha}$-value equal to $1.896 \mathrm{MeV}$ and $\ell=0$, or $\left.i i\right)$ to the ground state of the daughter, in this latter case with $Q_{\alpha}=1.987 \mathrm{MeV}$ and $\ell=2$. The $Q_{\alpha^{-}}$ values here referred include a small, but important, correction due to the screening to the nucleus caused by the surrounding electrons [6]. The alpha decay model we have proposed, which is semiempirical in nature, has a unique adjustable parameter, $0<g \leq 2 / 3$, which parameter-value is determined from decay data for known alpha transitions [68]. Parameter $g$ is related to the penetrability factor through the overlapping region of nascent fragments or, in other words, to the "arrival" of the alpha particle at the nuclear surface, i.e. the alpha particle preformation probability (see [6] for details). 
We used into the model the mass-excess, half-life, and alpha branching ratio values reported by Audi et al. [11] for the two ${ }^{147,148} \mathrm{Eu}$ isotopes to obtain for the alpha transitions of $\ell=0$ under assumption $i$ ) above a value $g=0.010$. On the other hand, a systematic study of the alpha decay half-lives using the same approach, but over 336 alpha emitter nuclides, has given $g=0.122$ [8]. Therefore, considering the transitions of $\ell=0$ one obtains two sets of half-life evaluations, one for each value of $g$. Results are depicted in figure 1 as a Geiger-Nuttall-like plot. The best agreement between calculated and measured values is found with parameter-value $g=0.010$ (circles and dashed line). However, for the particular case of ${ }^{151} \mathrm{Eu}$ isotope a difference by a factor $\sim 5$ is still present, which seems to indicate that very probably the alpha transition for this isotope does not proceed with $\ell=0$.

Now, following assumption $i i$ ) above, the ground-state of ${ }^{151} \mathrm{Eu}$ isotope would decay to the ground state of the daughter ${ }^{147} \mathrm{Pm}$ with $Q_{\alpha}=1.987 \mathrm{MeV}$ and $\ell=2$ (this is because spin and parity are changed from $5 / 2^{+}$to $7 / 2^{+}$). In this case, experimental information on alpha decay of Eu isotopes is not available at all (decays from ${ }^{147,148} \mathrm{Eu}$ do not proceed with $\ell=2$; parameter $g$ results indeed in negative values). An estimation of $g$-value to be used in this case has been obtained, however, from $g$-values determined previously in our alpha decay systematics, namely, that for bismuth isotopes [6] $(g=0.385$ for $\ell=5$ transitions) and the one for all alpha decay cases of $\ell=0$ transitions [8] $(g=0.122)$. Next, a dependence of $g$ upon $\ell$ is assumed of the form

$$
g_{\ell}=g_{0}+k \ell(\ell+1)
$$

where $g_{0}=0.122$ and $k=0.877 \times 10^{-2}$ as they come from the $g$ - and $\ell$-values just mentioned. Assumption (1) means that the increasing of centrifugal effects is directly related to an increasing in the overlapping effects, i.e. to a lowering of the preformation probability. Stated another way, when a change of spin and parity occurs $(\ell \neq 0)$, not only the external barrier (coulomb plus centrifugal) is more difficult of being penetrated, but also the internal (nuclear plus inertial effects) barrier. Equation (1) thus gives $g=0.175$ for $\ell=2$. Inserting these two values into the half-life routine calculation [6] one obtains $T_{1 / 2}=8.5 \times 10^{18} \mathrm{yr}$ for ${ }^{151} \mathrm{Eu}$ isotope, which value agrees quite completely with both 
the observed $\left(5_{-3}^{+11} \times 10^{18} \mathrm{yr}[3,4]\right)$ and the theoretical, calculated value of $3.6 \times 10^{18} \mathrm{yr}$ as reported in [4]. Regarding ${ }^{150} \mathrm{Eu}$ isotope, for which case one can have $\ell=2$ too, the evaluated partial alpha decay half-life amounts to $\sim 2.7 \times 10^{14} \mathrm{yr}$. Results are plotted in figure 1 (triangles and full line) from which, by a simple extrapolation procedure, one has $\sim 10^{28}$ yr and $\sim 10^{135}$ yr for the estimated partial alpha-decay half-life values for ${ }^{152} \mathrm{Eu}$ and ${ }^{153} \mathrm{Eu}$ isotopes, respectively. In both cases there is clearly no chance to detect any alpha activity at all.

To conclude, ${ }^{\text {nat }} \mathrm{Eu}$ has been demonstrated to exhibit also a rare alpha activity ( $\sim 30$ disintegrations/h-kg), in addition to natural Bi ( 12 disintegrations/h-kg) and tungsten ( $\sim 0.3$ disintegrations $/ \mathrm{h}-\mathrm{kg})$ previously detected $[10,12]$, this latter representing still a record.

\section{Acknowledgments}

The authors are thankful to Dr. Vladimir Tretyak for letting us know novel results on rare alpha radioactivity prior to publication.

\section{References}

[1] Audi G, Wapstra A H and Thibault C 2003 Nucl. Phys. A 729337

[2] Firestone R B 1996 Table of Isotopes 8th edn vol I (New York: Wiley)

[3] Tretyak V I 2007 Private communication

[4] Belli P et al. 2007 Nucl. Phys. A 78915

[5] Belli P, Cerulli R, Danevich F A, Grinyov B V, Incicchitti A, Kobychev V V, Laubenstein M, Nagorny S S, Tolmachev A V, Tretyak V I, and Yavetsky R P 2007 Nucl. Instrum. Methods Phys. Res. A 572734 
[6] Tavares O A P, Medeiros E L and Terranova M L 2005 J. Phys. G: Nucl. Part. Phys. 31129

[7] Tavares O A P, Terranova M L and Medeiros E L 2006 Nucl. Instrum. Methods Phys. Res. B 243256

[8] Medeiros E L, Rodrigues M M N, Duarte S B and Tavares O A P 2006 J. Phys. G: Nucl. Part. Phys. 32 B23

[9] Medeiros E L, Rodrigues M M N, Duarte S B and Tavares O A P 2006 J. Phys. G: Nucl. Part. Phys. 322345

[10] de Marcillac P, Coron N, Dambier G, Leblanc J and Moalic J P 2003 Nature (London) 422876

[11] Audi G, Bersillon O, Blachot J and Wapstra A H 2003 Nucl. Phys. A 7293

[12] Zdesenko Yu G, Avignone F T III, Brudanin V B, Danevich F A, Nagorny S S, Solsky I M and Tretyak V I 2005 Nucl. Instrum. Methods Phys. Res. A 538657

\section{Figure Captions}

Fig. 1 Geiger-Nuttall plot for Europium isotopes. Partial alpha-decay half-life, $T_{1 / 2_{\alpha}}$, is plotted (in log-scale) against the inverse square root of $Q_{\alpha}$-value for ${ }^{147-151} \mathrm{Eu}$ isotopes as indicated. The full circle and triangle for ${ }^{151} \mathrm{Eu}$ represent the observed value reported in [4], and the full circles for ${ }^{147,148} \mathrm{Eu}$ isotopes are data from [11]. The straight lines are least squares fits through calculated half-life values (open symbols) as explained in the text. 


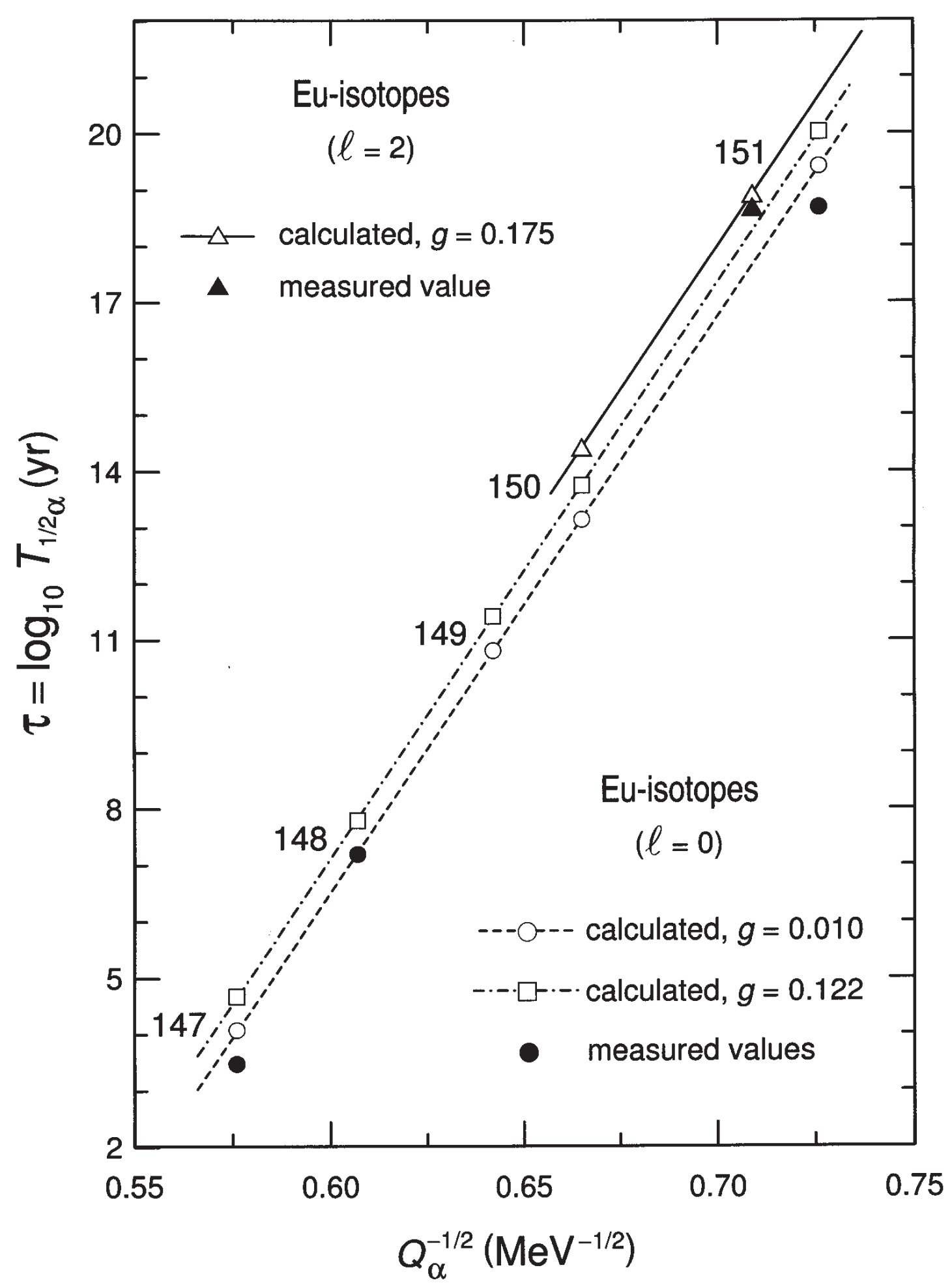

Figure 1 